Ľubor Kresák

Astronomical Institute, Slovak Academy of Sciences, Bratislava, Czechoslovakia

The attitude toward the systems of interplanetary objects is largely different from that accustomed in stellar astronomy. The stellar systems are viewed from the outside, with their shape, population, and internal structure apparent at first glance. The motions are difficult to recognize, if ever, and so the individual orbits are degraded to a background mechanism maintaining the observed organization and rotation of the system. In contrast to this, we dispose of copious information on the dynamics of individual interplanetary objects, but the bodies to which the orbital data refer are sampled under the influence of strong selection effects. Lacking a consistent survey of their systems, we tend to think of them in terms of the biased statistics in the phase-space of orbital elements, rather than in terms of the real three-dimensional distributions. No wonder that we often come across serious misrepresentations : the asteroid belt depicted as a plane ring divided by the Kirkwood gaps like the ring of Saturn; the Trojan clouds as small spherical systems resembling globular star clusters; or the meteor streams as elongated rings of uniform width and population all around. And we seem to receive these gross misrepresentations with surprisingly little annoy ance.

The aim of the present analysis was just to reconstruct the outer aspect of the systems of minor planets and comets, using all orbital data as available by the end of 1977, and attempting to eliminate the observational biases. The mein results are show in Figures 1 and 2 , in the form of smoothed equidensity plots in a plane passing through the Sun perpendicular to the ecliptic. The equidensity numbers $Q$ are logarithms with base two of the reciprocal mean local densities. Hence, an increase of $Q$ by one indicates a reduction of the density to one half, and an increase by three (solid lines) indicates a doubling of the mean separation of the objects. The unit density, $Q=0$, is defined by the whole population uniformly dispersed within a volume of 1 A.U.3; a north-south symmetry is assumed throughout. The cylindrical co-ordinates used are $X=R \cos B, Z=R \sin B$, L. Since the structure of the systems is mainly controlled by Jupiter, averaging of the equidensity contours in the $X, Z$-plane and referring the pattern to the mean heliocentric distance of Jupiter makes some features, depending on $\mathrm{L}$, disappear. These are : 1 . The effect of inclination of 
Jupiter's orbit to the ecliptic, smearing out the density pattern by \pm $0.023 \mathrm{X}$ in $\mathrm{Z}$. This can be neglected at the resolution used. 2 . The effect of eccentricity of Jupiter's orbit, smearing out the pattern by $\pm 0.048 \mathrm{X}$ in $X$. Since, due to the law of areas, the radius vector of an eccentric ring with one focus at the Sun is proportional to the square root of the population in $L$, the eccentricity of each system can be evaluated from the longitude distribution. 3. Periodic perturbations by Jupiter, making some pronounced irregularities revolve around the Sun with the planet. The extreme example, the Trojan clouds, has been omitted from the present analysis and will be discussed elsewhere.

\section{MINOR PLANETS}

The section through the asteroid belt, as plotted in Figure 1 , above, is a composite picture. Its inner region, up to $Q=10$, refers to the 121 largest asteroids, with individual masses presumably exceeding $3 \times 10^{18} \mathrm{~kg}$. Their selection was based on the list of compositional types and diameters by Zellner and Bowell (1977) and absolute magnitudes by Gehrels and Gehrels (1977). Carbonaceous and anomalous dark asteroids were included down to a diameter of $130 \mathrm{~km}$; for silicaceous and other asteroids of higher reflectivity the limiting diameter was set $10 \%$ lower, to account for their presumably lower bulk densities. There are exactly 100 objects meeting these criteria, in the list of Zellner and Bowell. Using the observed proportion of different compositional types as a function of the revolution period, it was estimated that the objects for which no physical observations are available include about 21 additional carbonaceous objects and no silicaceous object above the adopted mass limit. Therefore, the 21 absolutely brightest asteroids of this group were added to the sample, making a total of 121 objects with a probable $4 \%$ share of wrong classifications. To map the outskirts of the belt, 76 outlying objects with masses presumably exceeding $3 \times 10^{15} \mathrm{~kg}$ were extracted from a complete list of objects with known orbits (Efemeridy Malykh Planet, Minor Planet Circulars) and elaborated in a similar way. Hence, the outer equidensities $(Q>10)$ are based on the statistics of objects of continually decreasing size. Some uncertainty in the density gradient is due to an implicit assumption of the mass distribution index, adopted as a compromise between the results of Zeliner and Bowell and those of the McDonald and Palomar-Leiden Surveys.

It is noteworthy that at $Q>7$ the thickness of the belt perpendicular to the ecliptic is even a bit greater than its width. Major deformations are evidently associated with the depletion by Earth, Mars, and Jupiter. The first outer boundary (I) applies to the circulating asteroids. The second (II), stretching all around except in the vicinity of Jupiter, is formed by the librators of Hilda type. It may be imagined as a horseshoeshaped extension of the belt near its central plane. The size of the whole system is determined by the carbonaceous objects, with the silicaceous population prevailing in a flat sunward core and essentially absent beyond $X=3.6$. The belt is markedly eccentric, with the major axis aligned to that of Jupiter and with nearly the same eccentricity, 0.05. At any time, $50 \%$ of the asteroids are situated within $2.56<X<3.20$ and $90 \%$ within 2.15 $<\mathrm{X}<3.66$; $50 \%$ within $-0.28<\mathrm{Z}<+0.28$ and $90 \%$ within $-0.91<\mathrm{Z}<+0.91$. 

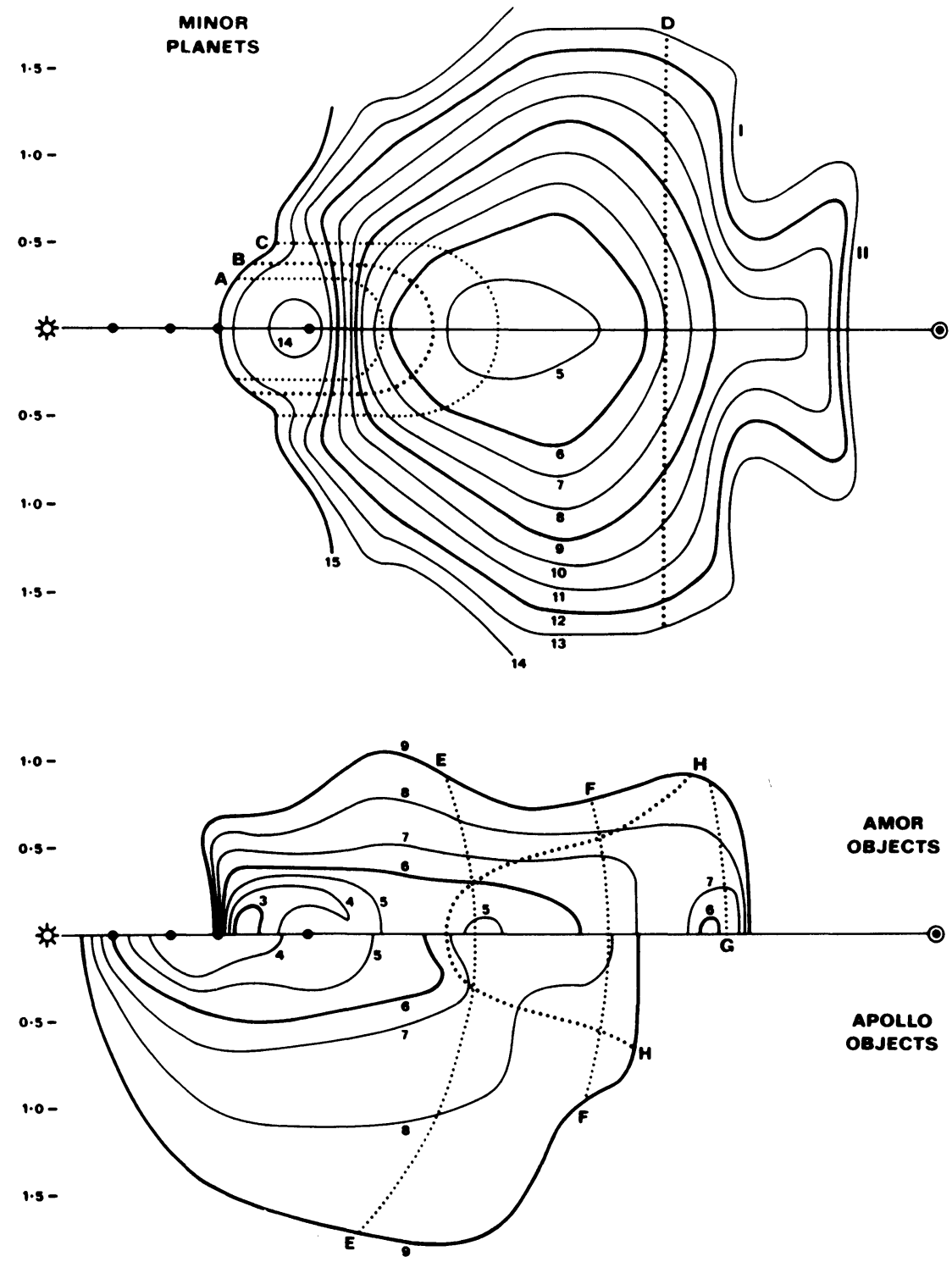

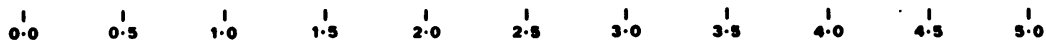

Figure 1. Equidensity contours of the main asteroid belt (above), and of the imbedded systems of the Amor and Apollo objects (below), in the plane passing through the Sun perpendicular to the ecliptic. Each step corresponds to a density ratio of $2: 1$, mean positions of the major planets are marked by solid dots. A, B, C, D - density ratios of $2: 1,1: 1,1: 2$, and 1:100 of the silicaceous to carbonaceous population. E, F, G - mean solar distances corresponding to the resonances of $1: 3,1: 2$, and $2: 3$ with Jupiter. H - density ratio of $1: 1$ of the Amor to Apollo objects. 


\section{AMOR AND APOLLO OBJECTS}

With two exceptions, the Amor and Apollo objects fall short of the size limit adopted for determining the contours of the main asteroid belt. Therefore, a separate analysis was based on the orbits of all such objects known by the end of 1977 : 18 of Amor type $(1<q<1.25$ ) and 25 of Apollo type ( $q<1$, but including 1580 Betulia and 1917 Cuyo). As the discoveries of these objects are seriously biased by observational selection, subsamples of different absolute brightness were formed to extrapolate to complete counts.

The Amor objects exhibit a steep decline of the density towards the Earth's orbit and, on the other side of a sharp concentration, a zone of moderate depletion around the orbit of Mars. Secondary maxima appear at heliocentric distances corresponding to the $1: 3$ and $2: 3$ circular resonances with Jupiter, the latter being especially pronounced. The system is rather flattened and markedly aligned to the apsides of Jupiter, $\theta=0.13$; the density gradient is similar to that of the main asteroid belt. The Apollo objects exhibit a flat maximum in the region of terrestrial planets. An abrupt change of the density gradient near $Q=7$ suggests the presence of two components : a thinner core, akin to the Amor population but concentrated within the $1: 3$ resonance mark, and a thick diffuse halo. Only six of the currently known objects, including those with nearly cometary values of the Jacobian constant in the system Sun-Jupiter, can be definitely classifled as belonging to the halo. Thus the three-dimensional distribution of the Apollo objects lends indirect support to the assumption that some of them may be extinct cometary nuclei. Calibration of the equidensity curves by the figures obtained for the Earth's vicinity (Kresák, 1978) suggests that there exist, in all, about 250 Apollo objects and 150 Amor objects with diameters exceeding $1 \mathrm{~km}$. However, these may be slight underestimates if the system includes a significant proportion of objects of very low reflectivity (cometary nuclei ?)

\section{CONETS}

The long-period comets exhibit a mean density of about 0.09 A.U. -3 throughout the inner solar system (Kresák and Pittich, 1978). They form a uniform background which is enhanced around the ecliptical plane and, in particular, near the orbit of Jupiter, by the comets revolving in shortperiod orbits. A complete list of comets of $\mathrm{P}<20$ years, which are currently under observation, includes 70 objects; an additional 23 objects can be regarded as lost. The available sample of orbits (Marsden, 1975, and other sources) is seriously biased by observational selection, mainly on account of the considerable brightness changes of comets with heliocentric distance. To eliminate or, at least, mitigate the bias, two lines of evidence were used : 1. Comparison of the total sample with a subsample of 27 presumably largest objects, nomely those observable beyond $R=3$ A.U. 2 . An empirical relation between the perihelion distance and the discovery probability, as established earlier for the long-period comets. The degree of reliability of the density contours obtained is conceivably lower than for the asteroids; nevertheless, the general pattern should be correct. 

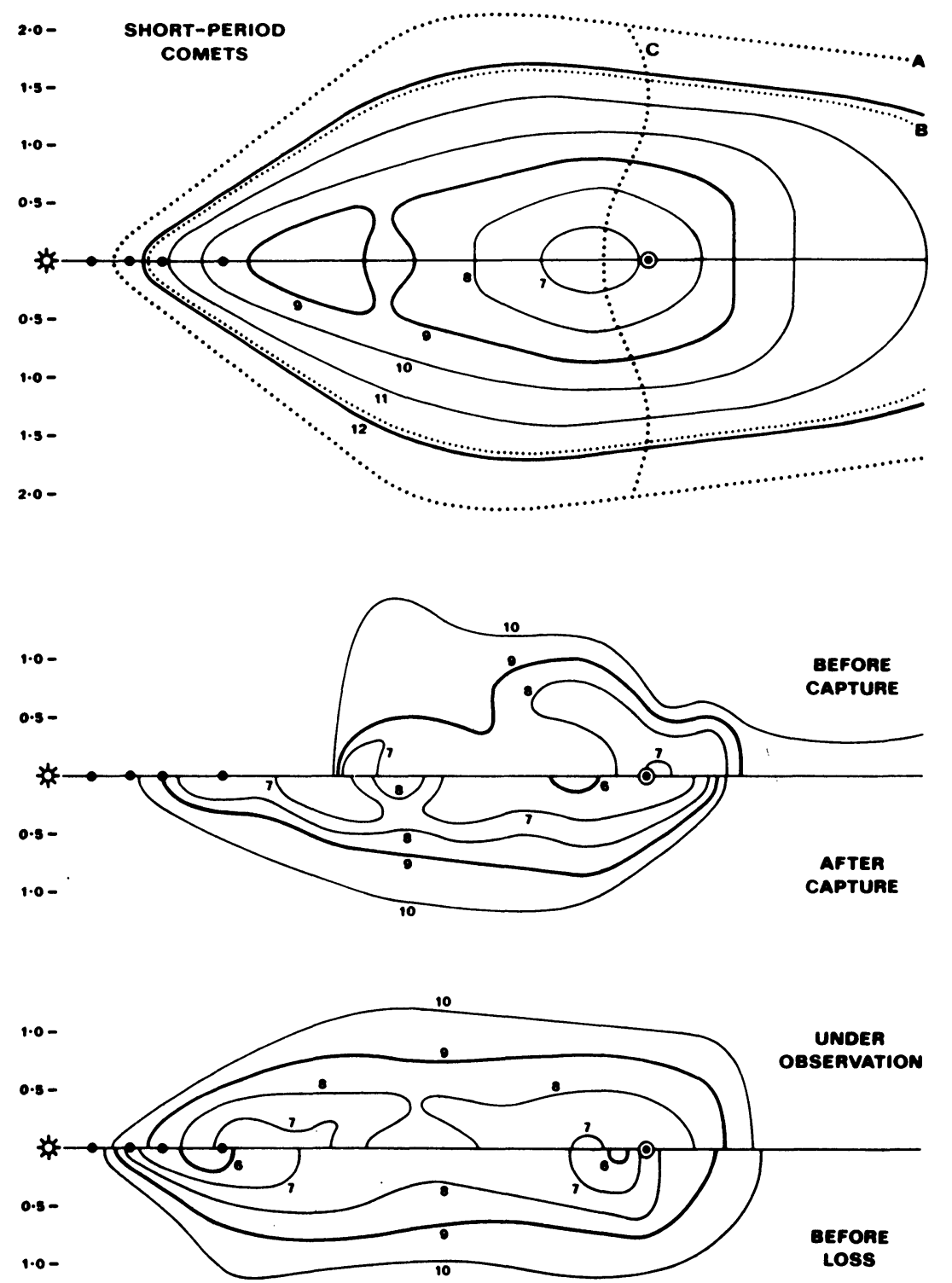

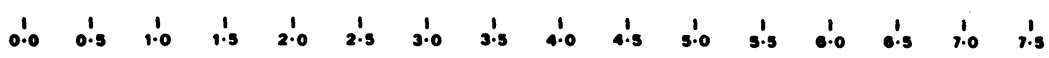

Figure 2. Equidensity contours of the system of short-period comets, $P<$ 20 years (above), and the flow of the observed objects through the system (below). Notation is the same as in Figure 1, only the scales are reduced by a factor of 2/3. A - density equals to the uniform background of longperiod comets; B - the same for nuclear diameters exceeding $1 \mathrm{~km}$; C - density equal that of the main-belt asteroids with diameters exceeding $1 \mathrm{~km}$. 
The population of the wole system, depicted in its quasi-steady state in Figure 2 above, undergoes frequent changes. While the perturbational displacement of a given object is equally probable in both directions, towards the Sun and away from it, the presence of an outer source (Oort's cloud) and the progressive disintegration (at a rate depending on perihelion distance) tends to separate the objects by age, with physically older and deactivated comets preferring the sunward region of the belt. The only irregularity wich appears statistically significant, is a moderate depletion near $X=2.8$ to $3.0, Z=0$. This may be due to the fact that newly captured objects, with perihelia in this zone and aphelia close to Jupiter, enter unstable orbits of approximate resonance $2: 3$ and are subject to repeated strong perturbations three revolutions later.

The distribution patterns preceding and following the capture by Jupiter refer to the same 17 objects which experienced an encounter at $q>$ 2.5 and were discovered, within the next two revolutions, in orbits of substantially reduced perihelion distance ( $\Delta q=-1.0$, on the average). Some of the precapture orbits were kindly supplied, in advance of publication, by N.A. Belyaev, B.G. Marsden, E. Pittich, and H. Rickman. The precapture orbits obviously pass close to Jupiter, and the oblique extension protruding from the area of maximum concentration is due to their orbital inclinations. After the capture, the main concentration passes from outside to inside the orbit of Jupiter and a secondary maximum, produced by the perihelia situated near the other node, appears. At the same time, a distinct alignment to the apsides of Jupiter is built up, with an effective eccentricity of 0.20 . The pattern for the 70 comets under observation, in their current osculating orbits, shows similar features; only the maximum, no longer coinciding with Jupiter in longitude, becomes less pronounced, and the effective eccentricity drops to 0.15 . The last pattern refers to the 23 lost comets at their last observed apparitions. Although this does not necessarily mean the final deactivation, a shift towards the Sun is apparent. Another characteristic feature is a sharp concentration just within the orbit of Jupiter.

The relative densities calibrated by the absolute near-earth data (Kresák, 1978) again permit us to estimate the total number of the objects. The result is 300 active, or potentially active, comets with nuclear diameters exceeding $1 \mathrm{~km}$, and 600 to 700 smaller ones, moving in orbits of $P$ $<20$ years at any time. For comparison, the total number of long-period comets situated at any time within the heliocentric distance of Jupiter is about 50 . In the region of their maximum concentration, short-period comets outnumber the long-period comets by a factor of 100; but anywhere beyond 2 A.U. from the ecliptical plane the long-period comets prevail.

\section{RE FERENCES}

Kresák, L.: 1978, "Bull. Astron. Inst. Czechosl." 29, pp. 114-125. Kresák, L., Pittich, E.M.: 1978, "Bull. Astron. Inst. Czechosl.", in press. Marsden, B.G.: 1975, "Catalogue of Cometary Orbits", SAO Cambridge. Zellner, B., Bowell, E.: 1977, in A.H. Delsemme (ed.), "Comets, Asteroids, Meteorites", IAU Coll. 39, Univ. Toledo, pp. 185-197. 